\title{
FACTORES NO GENÉTICOS QUE AFECTAN LA PRODUCCIÓN Y COMPOSICIÓN DE LA LECHE EN UN REBAÑO DE PARICIONES BIESTACIONALES EN LA DÉCIMA REGIÓN DE LOS LAGOS, CHILE
}

\section{Non genetic factors affecting milk production and composition in a dairy herd with two calving seasons in Los Lagos Region, Chile}

\author{
Lucio Pérez P. ${ }^{1}$, René Anrique G. ${ }^{2}$, Humberto González V. ${ }^{3}$
}

\begin{abstract}
A B S T R A C T
A total of 1,578 lactations of 493 cows were analyzed between 1990 and 2001. Lactations were grouped according to calving season (autumn and spring), month of calving within season (autumn: March, April, May; spring: July, August, September), cow age, number of calvings and lactation year. Accumulated production up to 305 days was considered; shorter lactations were not adjusted in order to detect environmental effects on the production period. Monthly milk production was adjusted to 30 days and standardized at $4 \%$ fat content. Variables evaluated were milk production, production and concentration of protein and fat, lactation length and persistency. Independent variables were analyzed estimating average minimum squares. With autumn calvings, milk production, fat production and lactation persistency were $5,293 \mathrm{~kg}, 205 \mathrm{~kg}$ and $72.1 \%$, respectively, being greater $(\mathrm{P} \leq 0.05)$ than spring calvings $(5,114 \mathrm{~kg}, 198 \mathrm{~kg}$ and $66.2 \%)$ with no difference in protein production. During the first half of lactation, milk production was lower with autumn calvings and the opposite was observed in the second half of lactation, indicating in both cases an underutilization of production potential of the cows for nutritional reasons. Lactation yield increased with earlier calvings in both seasons $(5,600$ vs. $5,440 \mathrm{~kg}$ in March and May with autumn lactations; and 5,303 vs. $4,980 \mathrm{~kg}$ in July and September with spring lactations). Milk protein content was greater $(\mathrm{P} \leq 0.05)$ with spring lactations compared to autumn lactations (3.23 vs. $3.19 \%$, respectively) with no differences in milk fat content (3.72 vs. $3.71 \%)$.
\end{abstract}

Key words: calving season, lactation curves, milk production and composition.

\author{
R E S U M E N
}

Se analizaron 1.578 lactancias de 493 vacas generadas entre 1990 y 2001. Se agruparon por época de parto (otoño, primavera), mes de parto dentro de época (otoño: marzo, abril y mayo; primavera: julio, agosto, septiembre), edad, número del parto, año de inicio de lactancia. Se consideró producción acumulada hasta 305 días; lactancias menores no se proyectaron para detectar efectos ambientales sobre el período de producción. La producción mensual se corrigió a 30 días y se estandarizó a $4 \%$ de materia grasa. Se evaluó producción de leche, producción y concentración de proteína y grasa, duración y persistencia de lactancia. Las variables independientes se analizaron estimando promedios mínimos cuadrados. Con partos de otoño, las producciones de leche, de grasa y la persistencia, respectivamente, $5.293 \mathrm{~kg}, 205 \mathrm{~kg}$ y $72,1 \%$, fueron superiores $(\mathrm{P} \leq 0,05)$ que con partos de primavera $(5.114$ $\mathrm{kg}, 198 \mathrm{~kg}$ y 66,2\%), sin diferencias en producción de proteína. En la primera mitad de lactancia, la producción fue menor con partos de otoño y lo contrario ocurrió en la segunda mitad, indicando en ambos casos una subutilización del potencial productivo por razones nutricionales. La producción por lactancia aumentó con partos tempranos dentro de época (5.600 vs $5.440 \mathrm{~kg}$ en marzo y mayo, con lactancias de otoño; y 5.303 vs $4.980 \mathrm{~kg}$ en julio y septiembre, con lactancias de primavera). El contenido de proteína fue mayor $(\mathrm{P} \leq 0,05)$ en lactancias de primavera que de otoño $(3,23$ vs $3,19 \%)$ sin diferencias en grasa láctea $(3,72$ vs $3,71 \%)$.

Palabras clave: época de parto, curvas de lactancia, producción, composición de la leche.

\footnotetext{
${ }^{1}$ Ferosor Agrícola, Los Carrera 643, Osorno, Chile. E-mail: lperez@ferosor.cl

${ }^{2}$ Universidad Austral de Chile, Facultad de Ciencias Agrarias, Casilla 567, Valdivia, Chile.E-mail: ranrique@uach.cl*Autor para correspondencia. ${ }^{3}$ Universidad de Chile, Facultad de Ciencias Agronómicas, Estación Experimental Oromo, Casilla 161, Purranque, Chile. E-mail: hgonzale@uchile.cl El resumen de este trabajo fue presentado en el XXVIII Congreso de la Sociedad Chilena de Producción Animal (Sochipa A.G.) realizado en la Universidad Católica del Maule, Talca, Chile, del 15-17 de octubre de 2003.

Recibido: 30 de marzo de 2005. Aceptado: 27 de junio de 2005.
} 


\section{INTRODUCCIÓN}

Las explotaciones lecheras de la zona sur de Chile (Regiones IX y X), generan más del 70\% de la producción nacional de leche recepcionada en planta. En éstas, los partos se concentran principalmente en primavera y otoño, con el objeto de satisfacer la exigencia de la industria orientada a lograr un abastecimiento uniforme a través del año. Existen, no obstante, muy pocas explotaciones con partos exclusivamente en primavera, las que corresponden en su mayoría a pequeños productores (Anrique et al., 1999).

El sistema de parición estacional de primavera se sustenta en la utilización de la pradera mediante pastoreo, ajustando los requerimientos nutricionales de las vacas en lactancia a la curva de crecimiento de los pastos. Diferente es la estrategia seguida en el sistema de partos de otoño; en este caso, durante la primera mitad de la lactancia, la alimentación se basa en forraje conservado, principalmente ensilaje de pradera, más suplementos suministrados en patio de alimentación; la segunda mitad de la lactancia se desarrolla en pastoreo (Anrique et al., 2004).

En la zona sur, la producción de leche individual tiende a ser mayor en las lactancias iniciadas en otoño (Herrera, 1997; Bravo, 1998), hecho que se explica, al menos en parte, por la mayor longitud y persistencia de las mismas respecto de las que comienzan a fines de invierno. Estas últimas se ven afectadas, entre otros factores, por las condiciones desfavorables de disponibilidad y calidad nutricional de la pradera durante el verano, lo que se traduce en curvas de menor persistencia y duración (González y Magofke, 1994).

Cabe mencionar que otras investigaciones también dan cuenta de variaciones estacionales en la composición láctea (Letelier, 1998; Pinto et al., 1998). La concentración de proteína tiende a ser mayor en las lactancias comenzadas en primavera, con respecto a las de otoño, lo que se debería a la alta disponibilidad y calidad de las praderas durante dicha época. Producto de este mismo hecho, se observa paralelamente una disminución en el tenor graso (O'Brien et al., 1997).

De lo antes expuesto, se desprende que al coexistir dos épocas de parición en una explotación lechera, se generan interrelaciones que influyen en las curvas de lactancia y en la producción y composición de la leche que son importantes de identificar y cuantificar. Esto permitiría orientar las estrategias de manejo tendientes a mejorar la eficiencia productiva y económica de estos sistemas.

El objetivo del presente estudio fue evaluar cuantitativamente el efecto de diferentes factores no genéticos, principalmente época y mes de parto, sobre la producción y composición láctea en un rebaño de parición biestacional, utilizando información recopilada en un período de 11 años.

\section{MATERIALES Y MÉTODOS}

Se analizó la información obtenida de 493 vacas Frisón Negro pertenecientes al rebaño lechero de la Estación Experimental Punahue de la Universidad Austral de Chile, Los Lagos, Provincia de Valdivia, X Región ( $39^{\circ} 51^{\prime}$ lat. Sur, $72^{\circ} 36^{\prime}$ long. Oeste, 230 m.s.n.m.). La zona es de clima templado lluvioso con influencia mediterránea y una precipitación media anual de 2.000 a $2.300 \mathrm{~mm}$.

Durante los meses de invierno, las vacas paridas en otoño se mantuvieron confinadas en un patio de alimentación, separadas en cinco grupos: $>25 \mathrm{~L}$ día $^{-1} ;<25$ y $>22$ L día $^{-1} ;<22$ y $>19$ L/día, más un grupo de primera parición. La base alimenticia la constituyó ensilaje directo de pradera más un aporte diferencial de concentrado, que osciló entre 2 y $7 \mathrm{~kg} \mathrm{~d}^{-1}$, de acuerdo al nivel de producción del grupo. Desde septiembre en adelante la alimentación se basó en la utilización de praderas, constituidas principalmente por trébol blanco (Trifolium repens L.) y ballica inglesa (Lolium perenne L.), mediante pastoreo rotativo, más una suplementación de concentrado según producción entre 2 y $5 \mathrm{~kg} \mathrm{~d}^{-1}$. Adicionalmente, se contempló una suplementación estival con cultivos forrajeros tales como lupino ( $\mathrm{Lu}$ pinus angustifolius L.) o alfalfa (Medicago sativa L.). El consumo de concentrado fluctuó entre 0,23 y $0,29 \mathrm{~kg} \mathrm{~L}^{-1}$ de leche.

Se emplearon 1.578 lactancias, desarrolladas durante 11 temporadas, transcurridas entre los años 1990 a 2001. Éstas se agruparon de acuerdo a época de parto (otoño o primavera), mes de parto dentro de época (otoño: marzo, abril y mayo; primavera: julio, agosto y septiembre), edad de la vaca, número ordinal de parto y año de inicio de la lac- 
tancia. En una primera etapa se eliminaron lactancias menores a 200 días, que constituyeron un $5,5 \%$ del total. En lactancias cuya duración excedió los 305 días $(60,3 \%)$ se consideró sólo la producción acumulada dentro de este período. El resto $(34,2 \%)$ no se proyectó a 305 días de duración, puesto que hacerlo impediría detectar efectos ambientales cuya principal manifestación se refleja en el largo del período de producción (García et al., 1984; 1987; González y Magofke, 1994; García et al., 1999).

Las producciones mensuales se ajustaron a una base común de 30 días y se estandarizaron a un contenido de $4 \%$ de materia grasa según la ecuación de Gaines y Davidson, citados por Lasley (1982). La persistencia de las curvas de lactancia se calculó de acuerdo a la ecuación desarrollada por Tapia et al. (1964), citados por Magofke et al. (1984):

$$
I P=\left(\sum_{i=1}^{10} \frac{\boldsymbol{x}_{i}}{\boldsymbol{x}_{\text {máx }}}\right) \times 10
$$

donde, $I P=$ índice de persistencia expresado en porcentaje; $\boldsymbol{x}_{i}=$ producción de leche en el $i$ ésimo mes de lactancia; $\boldsymbol{x}_{\text {máx }}=$ producción de leche del mes de máxima producción.

Las variables dependientes estudiadas fueron producción de leche sin corregir y estandarizada (4\% de materia grasa), producción y concentración de grasa y proteína láctea, duración y persistencia de la lactancia.

Preliminarmente, los efectos de la época y el número de parto se estimaron mediante el siguiente modelo estadístico:

$$
y_{i j k l m}=\mu+p_{i}+m_{j}\left(p_{i}\right)+a_{k}\left(p_{i}\right)+n_{l}+e_{i j k l m}
$$

donde, $y_{i j k l m}=$ variables dependientes; $\mu=$ promedio general; $p_{i}=$ efecto asociado a la época de parto $i ; m_{j}\left(p_{i}\right)=$ efecto asociado al mes de parto $j$ dentro de la época $i ; a_{k}\left(p_{i}\right)=$ efecto asociado al año de parto $k$ dentro de la época $i ; n_{l}=$ efecto asociado al número de parto $l ; e_{i j k l m}=$ error experimental.

Este análisis permitió determinar la significancia del mes y año de parto anidados dentro de cada época. Por este motivo, fue necesario usar un segundo modelo para efectuar comparaciones dentro de cada una de ellas. Sin embargo, el análisis precedente otorgó información referida a la comparación global entre otoño y primavera.

En una segunda etapa los análisis dentro de otoño y primavera se realizaron con el modelo descrito a continuación:

$$
y_{i j k l}=\mu+m_{i}+a_{j}+n_{k}+e_{i j k l}
$$

donde, $y_{i j k l}=$ variables dependientes; $\mu=$ promedio general; $m_{i}=$ efecto asociado al mes de parto $i$; $a_{j}=$ efecto asociado al año de parto $j ; n_{k}=$ efecto asociado al número de parto $k, e_{i j k l}=$ error experimental.

En ambas circunstancias, los modelos definitivos se estructuraron a partir de un conjunto de análisis previos que sirvieron para definir los niveles empleados en las variables independientes, y determinar si las interacciones de segundo grado entre los efectos principales eran significativas. Éstas no fueron incluidas debido a que no alcanzaron significancia estadística $(\mathrm{P}>0,05)$.

El análisis de las variables independientes que inciden sobre los distintos parámetros productivos, se realizó estimando promedios mínimos cuadrados, debido a que las subclases contenían un número desigual de observaciones. La obtención de éstos se realizó mediante el procedimiento GLM del Sistema de Análisis Estadístico SAS. Las comparaciones entre medias se efectuó empleando la prueba " $t$ " para promedios pareados (SAS Institute, 1992).

\section{RESULTADOS}

Los promedios mínimos cuadrados de las 1.600 lactancias, no ajustadas a 305 días para producción de leche sin corregir y producción de leche estandarizada, fueron de 5.510 y $5.256 \mathrm{~kg} \mathrm{vaca}^{-1}$, respectivamente. Las producciones y concentraciones de materia grasa y proteína fueron de 203 y $173 \mathrm{~kg}$ y de 3,71 y $3,21 \%$, respectivamente. La persistencia de la curva de lactancia en promedio alcanzó 72,9\%.

La edad media del rebaño fue de 4,8 años, la edad al primer parto de 2,5 años y el número de partos por vaca fue de 3,2. El largo de lactancia presentó una amplia dispersión, siendo los valores extremos 
de 150 y 650 días. El promedio real, sin corregir de esta variable fue de 333 días, mientras que para el período seco se obtuvo una media de 70 días. Debido a que las lactancias se cortaron a 305 días, los promedios mínimos cuadrados (Cuadro 1) difieren del valor antes señalado.

\section{Época de parto}

El Cuadro 1 presenta los resultados obtenidos al comparar globalmente diferentes variables productivas en lactancias iniciadas en otoño y primavera. Se observa que las vacas paridas en otoño superaron en producción de leche a aquellas que iniciaron su lactancia en primavera $(\mathrm{P} \leq 0,05)$, con una diferencia de $179 \mathrm{~kg}$ en favor de las primeras. Si bien el largo de la lactancia no difirió entre épocas, la persistencia fue mayor en 5,9 unidades porcentuales en los partos de otoño. En producción de grasa se observó una tendencia similar a la descrita para producción láctea, debido a que su concentración no varió entre épocas. En este caso, las lactancias iniciadas en otoño fueron un 3,6\% superior. E1 contenido proteico fue mayor con los partos de primavera, sin embargo, la producción de proteína no difirió entre épocas.

Al comparar las curvas de lactancia correspondientes a cada época de parto (Figura 1), se aprecia en ambos casos un desarrollo diferente. En la primavera, la producción se elevó levemente a partir del primer mes, para alcanzar un máximo de $21,9 \mathrm{~kg}$ día $\mathrm{a}^{-1}$ durante el segundo mes, y posteriormente, la curva declina en forma sostenida. En el otoño, la producción máxima $\left(22,4 \mathrm{~kg} \mathrm{~d}^{-1}\right)$ ocurrió durante el primer mes, superando en magnitud el nivel alcanzado por las curvas de primavera en igual período y la producción disminuye hasta el quinto mes de lactancia. Posteriormente la producción sube hasta el sexto, superando a los dos meses anteriores, para luego declinar en forma sostenida. La comparación de ambas curvas, ordenadas de acuerdo a mes calendario (Figura 1a), permite advertir que esta etapa de la lactancia de otoño es coincidente con la de mayor producción en las vacas de primavera, período que corresponde con la mayor disponibilidad de forraje, lo que explica en parte, la situación descrita.

Al comparar ambas curvas de lactancia de acuerdo al mes posparto (Figura 1b), se observa un comportamiento diferente. Las mayores producciones durante la primera mitad de la lactancia corresponden a las pariciones de primavera, situación que se invierte en la segunda mitad de la misma.

\section{Mes de parto dentro de época}

El efecto del mes de inicio de la lactancia en la época de otoño, se presenta en el Cuadro 1. La mayor producción de leche se obtuvo con partos en marzo, superando en $312 \mathrm{~kg}$ el promedio de la época y en 137 y $158 \mathrm{~kg}$ la producción observada con partos en abril y mayo, respectivamente. Si bien no

Cuadro 1. Efecto de la época y del mes de parto en la producción y composición de la leche.

Table 1. Effect of calving season and month of calving on milk production and composition.

\begin{tabular}{lccccccc}
\hline & $\begin{array}{c}\text { Leche 4\% MG } \\
(\mathbf{k g})\end{array}$ & $\begin{array}{c}\text { Grasa } \\
(\%)\end{array}$ & $\begin{array}{c}\text { Proteína } \\
(\%)\end{array}$ & $\begin{array}{c}\text { Grasa } \\
(\mathbf{k g})\end{array}$ & $\begin{array}{c}\text { Proteína } \\
(\mathbf{k g})\end{array}$ & $\begin{array}{c}\text { Largo de } \\
\text { lactancia } \\
(\mathbf{d i ́ a s})\end{array}$ & $\begin{array}{c}\text { Persistencia } \\
(\%)\end{array}$ \\
\hline $\begin{array}{l}\text { Época de parto } \\
\text { Otoño }\end{array}$ & $5.292 \mathrm{a}$ & $3,72 \mathrm{a}$ & $3,19 \mathrm{a}$ & $205,0 \mathrm{a}$ & $169,2 \mathrm{a}$ & $297 \mathrm{a}$ & $72,1 \mathrm{a}$ \\
$\quad \begin{array}{l}\text { Primavera } \\
\text { Otoño }\end{array}$ & $5.113 \mathrm{~b}$ & $3,71 \mathrm{a}$ & $3,23 \mathrm{~b}$ & $197,6 \mathrm{~b}$ & $172,3 \mathrm{a}$ & $296 \mathrm{a}$ & $66,2 \mathrm{~b}$ \\
$\quad$ Marzo & $5.599 \mathrm{a}$ & $3,68 \mathrm{a}$ & $3,17 \mathrm{a}$ & $216 \mathrm{a}$ & $177 \mathrm{a}$ & $304 \mathrm{a}$ & $73,1 \mathrm{a}$ \\
$\quad \begin{array}{l}\text { Abril } \\
\text { Mayo }\end{array}$ & $5.462 \mathrm{~b}$ & $3,68 \mathrm{a}$ & $3,20 \mathrm{a}$ & $210 \mathrm{~b}$ & $170 \mathrm{~b}$ & $297 \mathrm{~b}$ & $72,6 \mathrm{a}$ \\
$\begin{array}{l}\text { Primavera } \\
\text { Julio }\end{array}$ & $5.440 \mathrm{~b}$ & $3,66 \mathrm{a}$ & $3,19 \mathrm{a}$ & $209 \mathrm{~b}$ & $173 \mathrm{ab}$ & $290 \mathrm{c}$ & $71,9 \mathrm{a}$ \\
$\quad \begin{array}{l}\text { Agosto } \\
\text { Septiembre }\end{array}$ & $5.303 \mathrm{a}$ & $3,70 \mathrm{a}$ & $3,19 \mathrm{a}$ & $205 \mathrm{a}$ & $178,5 \mathrm{a}$ & $302 \mathrm{a}$ & $68,5 \mathrm{a}$ \\
\hline
\end{tabular}

Letras distintas en las columnas indican diferencias significativas $(\mathrm{P} \leq 0,05)$ según prueba " $\mathrm{t}$ " para promedios pareados.

MG: Materia grasa. 


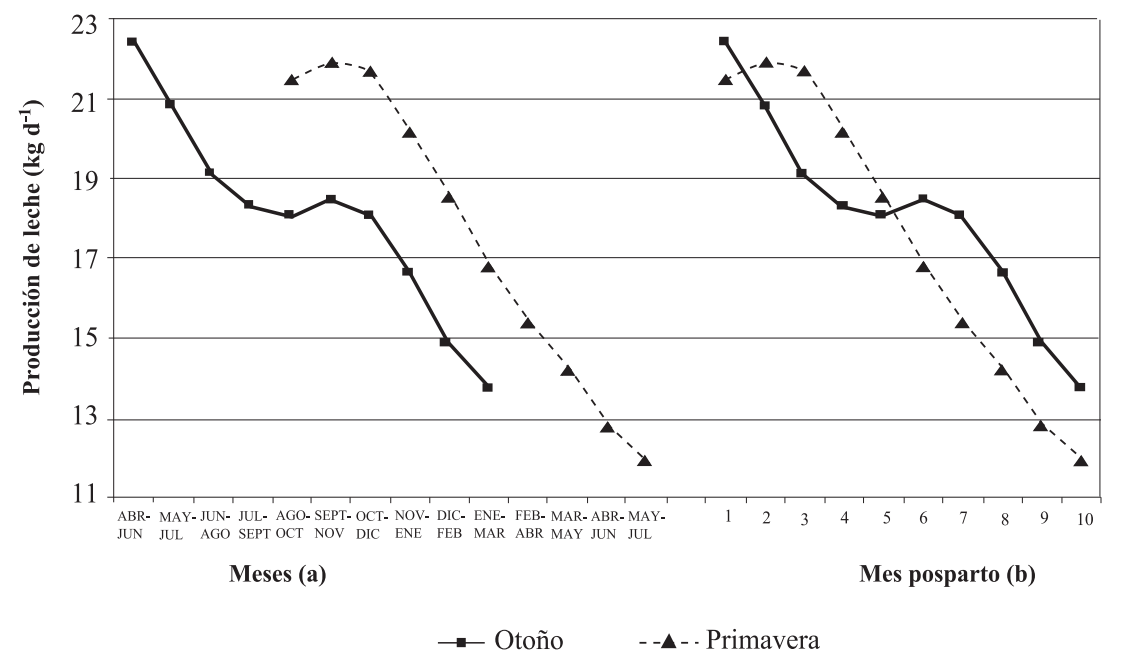

Figura 1. Curvas de producción de leche estandarizada iniciadas en otoño y primavera. Figure 1. Standardized milk production curves for autumn and spring lactations.

hubo diferencias significativas $(\mathrm{P}>0,05)$ entre lactancias iniciadas en abril y mayo, se manifestó una tendencia a menor producción asociada a lactancias iniciadas más tardíamente. La persistencia de la curva de lactancia no difirió significativamente entre los meses de parto, sin embargo, las lactancias iniciadas en marzo fueron, en promedio, 7 días más largas que las de abril y 14 días más largas que las de mayo.

Las concentraciones de grasa y de proteína no difirieron según el mes de inicio de la lactancia $(\mathrm{P}>0,05)$. La producción de grasa, sin embargo, fue mayor $(\mathrm{P} \leq 0,05)$ con partos de marzo, superando en 5,4 y $6,9 \mathrm{~kg}$ la observada en abril y mayo, respectivamente. Esta respuesta fue similar a la encontrada para la producción de proteína, que sólo fue superior $(\mathrm{P} \leq 0,05)$ con partos de marzo, sin diferencias $(\mathrm{P}>0,05)$ entre abril y mayo (Cuadro 1$)$.

El comportamiento de las curvas de lactancia de otoño, ordenadas según mes calendario y mes posparto (Figura 2), demuestra que la producción del primer mes de lactancia tiende a decrecer al atrasarse las pariciones desde marzo a mayo (Figura 2a). La producción en los primeros cuatro meses disminuye a una tasa similar, seguida de un aumento en la primavera, que es más marcado y ocurre antes, mientras más tardío es el parto (Figura 2b). Para vacas paridas en marzo, abril y mayo, el repunte se presentó al $7^{\circ}, 6^{\circ}$ y $5^{\circ}$ mes postparto, en el mismo orden, punto en el cual la producción de leche de las vacas paridas en mayo superó por 13,7 y 14,3\% a la de aquellas con parto en abril y marzo, respectivamente. La producción de leche una vez pasado el repunte de primavera, tiende a decrecer a una tasa más marcada con los partos más tardíos.

En partos de primavera (Cuadro 1) la producción por lactancia decreció al atrasarse los partos de julio a septiembre, sin diferencias significativas entre los meses de julio y agosto $(\mathrm{P}>0,05)$, con una diferencia de 133 y $323 \mathrm{~kg}$ al comparar lactancias de julio con lactancias de agosto y septiembre, respectivamente. Las vacas paridas en julio, en comparación con las de septiembre, produjeron un $6,1 \%$ más de leche, estuvieron 13 días más en lactancia y presentaron una desviación positiva de cinco unidades porcentuales en la persistencia de la curva. $\mathrm{Al}$ igual que en la época de otoño, los porcentajes de grasa y de proteína no difirieron significativamente según el mes de parto. Sin embargo, las cantidades de estos componentes lácteos respondieron de forma bastante similar, observándose las mayores producciones en lactancias iniciadas en julio y las menores en lactancias de septiembre.

El comportamiento de las curvas de lactancia iniciadas en primavera de acuerdo al mes de parto, ordenadas según mes calendario y mes posparto (Figura 3a) demuestra que la producción inicial (primer mes) igual que la producción máxima, aumentan al atrasarse el mes de parto, de julio a septiembre, seguido de una persistencia menor que hace decrecer la producción al avanzar la lactancia. Este efecto aumenta en magnitud en los partos 


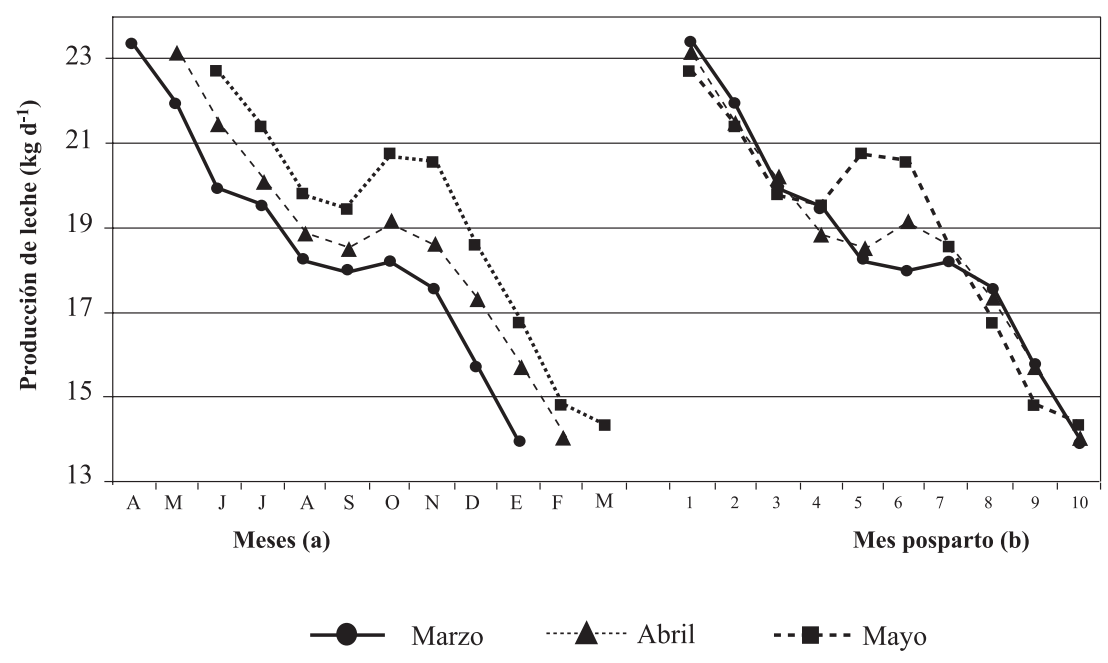

Figura 2. Curvas de producción de leche estandarizada iniciadas en marzo, abril y mayo.

Figure 2. Standardized milk lactation curves for lactations started in March, April and May.

más tardíos. En lactancias iniciadas en septiembre también se aprecia que las mayores producciones diarias $\left(23 \mathrm{~kg} \mathrm{~d}^{-1}\right)$ ocurrieron en los meses de octubre y noviembre, sin embargo, los niveles de producción en los meses restantes fueron inferiores a los alcanzados por las vacas paridas en julio y agosto (Figura 3b).

\section{DISCUSIÓN}

\section{Promedios mínimos cuadrados}

El nivel de producción del rebaño puede considerarse apropiado para el nivel de concentrado suministrado $\left(5\right.$ a $\left.7 \mathrm{~kg} \mathrm{~d}^{-1}\right)$. Comparativamente, el promedio por lactancia de los productores bajo control oficial es de $6.964 \mathrm{~kg}$ (Lama, J. 2004. Control Lechero Oficial. COOPRINSEM. Comunicación personal), con un largo de lactancia de 332 días. El contenido promedio de proteína y materia grasa láctea también es normal. Sin embargo, el largo promedio de lactancia real (333 días) es mayor a lo recomendado (Latrille, 1985), lo que refleja la existencia de algunos problemas reproductivos, cuyo análisis está fuera de los objetivos del presente estudio.

\section{Efecto de la época de parto.}

La mayor producción observada en lactancias de otoño comparado con lactancias de primavera es concordante con estudios previos (Herrera, 1997; Bravo, 1998) y se explica principalmente por la buena alimentación durante la segunda mitad de la lactancia, coincidente con el pastoreo de primavera, que favorece la persistencia.
La menor producción de leche observada con partos de otoño en relación a los de primavera, en la primera mitad de la lactancia (aprox. 15\%), tiene claras connotaciones de origen nutricional, la que se traduce en una limitante para la expresión del potencial productivo de las vacas en esta época (Figura $1 b)$. La mayor producción experimentada en la segunda mitad de la lactancia por las vacas de parto de otoño, sugiere una subutilización del potencial de las vacas de parto de primavera, por diferencias en la persistencia de la lactancia, las que también podrían ser atribuibles a una alimentación insuficiente (Bravo, 1998). En este sentido, es clara la desventaja de los partos de primavera, ya que la segunda mitad de la lactancia coincide con el período verano-otoño, en que la pradera es claramente de menor calidad que en los meses previos, reduciéndose la persistencia (Teuber y Balocchi, 2003).

El análisis de las curvas de lactancia (Figura 1a) da cuenta de la importancia de la pradera como fuente de alimento para el rebaño en estudio. En primavera, independiente del momento del parto, los mayores niveles productivos se alcanzaron en el mes de octubre. En los partos de otoño, la caída de los niveles de producción en el período de confinamiento (abril a agosto) se revirtió al salir las vacas a pastoreo, observándose, igual que con partos de primavera, un aumento de producción en octubre, que es el mayor nivel productivo de la segunda mitad de la lactancia de estas vacas. Esto sería en gran medida resultante 


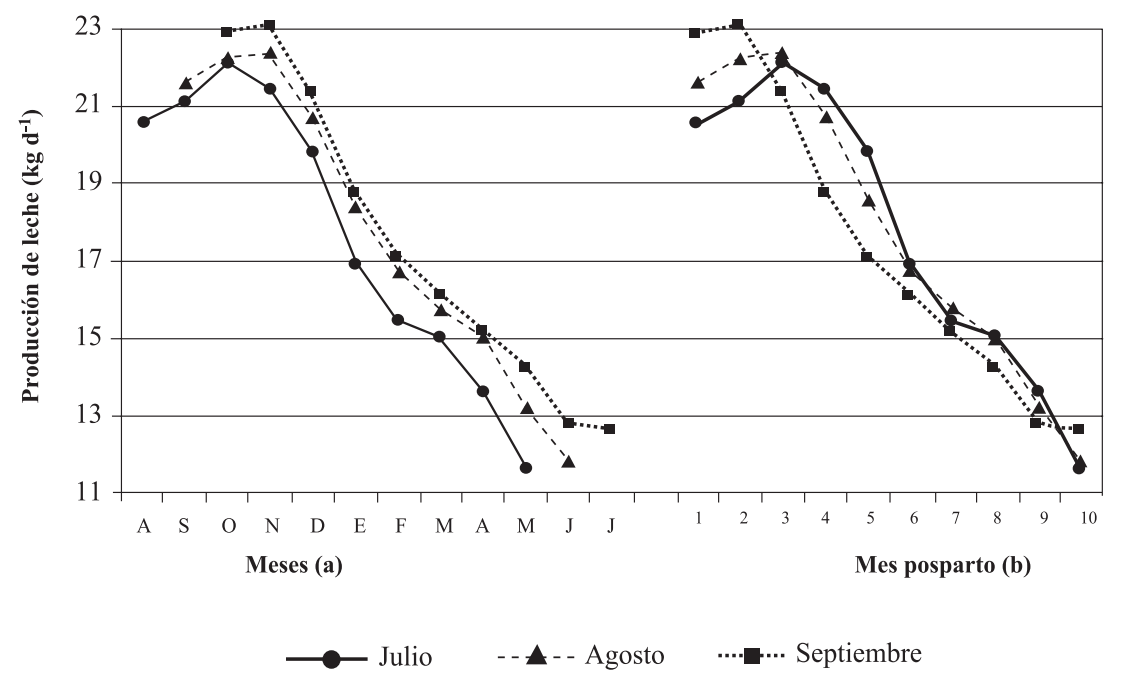

Figura 3. Curvas de producción de leche estandarizada iniciadas en julio, agosto y septiembre.

Figure 3. Standardized milk production curves for lactations started in July, August and September.

de un cambio nutricional determinado por la mayor calidad de la pradera en relación a la ración invernal y muy posiblemente por un mayor consumo relativo influido por la alta digestibilidad de la pradera. A su vez, la magnitud de este repunte es menor mientras más temprano es el parto, favoreciendo una mayor persistencia de la lactancia. Coincidentemente, Herrera (1997) y Bravo (1998), encontraron que las lactancias iniciadas en otoño se ven favorecidas en cuanto a su longitud en la primavera siguiente, en tanto que las lactancias iniciadas en primavera se ven acortadas por el período de verano. Sin embargo, el largo de la lactancia no difirió entre épocas en el presente estudio, por lo cual las diferencias de producción son principalmente atribuibles al efecto de la persistencia. Cabe señalar, no obstante, que aunque la superioridad de los partos de otoño es sólo de un 3\%, este efecto podría ser de mayor magnitud si se otorgaran a los animales mejores condiciones alimenticias en el primer tercio de la lactancia.

El mayor tenor de proteína observado en las lactancias de primavera comparadas con las de otoño, se manifestó más claramente en la primera mitad de la lactancia y en los últimos tres-cuatro meses de ésta, abarcando sobre el $80 \%$ del período total. Durante el "peak" de producción primaveral el tenor de proteína fue bastante uniforme independientemente de la época de parto. El mayor contenido de proteína en lactancias de primavera, es concordante con lo detectado a nivel de la industria leche- ra (Nestlé. 2004. Registros planta lechera Osorno. Comunicación personal). La producción de grasa respondió de igual forma que la producción de leche, siendo significativamente mayor en otoño (Cuadro 1). El alto contenido de fibra de los forrajes conservados, característicos del sur de Chile (Teuber y Balocchi, 2003), favorece la concentración de grasa láctea en los meses invernales. Sin embargo, la diferencia de concentración de este componente entre épocas no fue significativa $(\mathrm{P}>$ $0,05)$, debido a que efectos detectados en una parte de la lactancia, como es la depresión observada a comienzos de primavera, es contrarrestada por aumentos en el resto de la lactancia, debido al mayor nivel de fibra de los pastos (Latrille, 1993; González, 2002).

\section{Efecto del mes del parto}

La mayor producción por lactancia asociada a partos tempranos dentro de época (Cuadro 1), coincide con lo encontrado en otros estudios (Magofke $e t$ al., 1984; Cortés, 1986; García et al., 1987; Herrera, 1997; García et al., 1999).

Las vacas paridas en marzo se encuentran en su $10^{\circ}$ mes posparto en el mes de enero, mientras que las paridas más tarde cursan el último tercio de su lactancia en el verano (Figura 2a) y deben enfrentar las limitaciones de calidad y disponibilidad de la pradera, lo cual influye más negativamente en la producción. La diferencia de duración entre lactancias iniciadas en marzo y mayo fue de aproximadamente 15 días. También ha habido estudios en que 
la persistencia ha sido la principal causal de diferencias productivas al atrasarse el inicio de la lactancia (Herrera, 1997).

Es interesante resaltar el incremento en producción de leche ocurrido en primavera, luego de salir las vacas a pastoreo, con un "peak" en el mes de octubre, independientemente del mes de parto. La magnitud de este aumento de producción, que fue mayor en las vacas paridas más tardíamente, indica un mayor potencial de respuesta al aporte de la pradera en estas vacas, las cuales se encuentran en un estado más temprano de lactancia y por lo tanto, en ellas aún se prioriza la síntesis de leche por sobre la acumulación de reservas (Holmes et al., 2002).

Respecto de lactancias de primavera, las mayores producciones observadas con partos tempranos (Cuadro 1), son atribuibles al mayor sincronismo entre requerimientos y disponibilidad y calidad de la pradera. Por otra parte, el efecto de la sequía estival se atenúa, ya que las vacas de parto temprano llegan al período estival en una fase de declinación en su curva de lactancia (Figura 3a). En cambio, con parto de septiembre se llega al mes de enero con sólo cuatro meses de lactancia, estando afectas a una restricción nutricional más acentuada durante los meses de verano, lo cual determina lactancias de menor persistencia. Por ende, suplementar los animales durante este período puede mejorar la persistencia, sin embargo, difícilmente ello se va a traducir en una mayor longitud de la curva de lactancia. Como se puede apreciar, las fechas de parto son más variables que las fechas de secado, que están en gran medida determinadas por la disponibilidad de pradera, la cual disminuye fuertemente su crecimiento al ingresar al período invernal.

En lactancias de otoño, el decrecimiento de la producción inicial (primer mes) observado al atrasarse el mes de parto (Figura 2), se explicaría por la disminución progresiva en la disponibilidad de praderas desde marzo a mayo (Riveros y Magofke, 1983; Teuber y Balocchi, 2003), determinando menores índices de condición corporal y una menor utilización de reservas corporales al inicio de la lactancia (Latrille, 1985). Estos resultados, si bien no se pueden generalizar, señalan una situación muy posible de ocurrir si no se suministra una buena alimentación en los meses previos al parto de otoño, que coinciden con el período estival que normalmente es restrictivo. Al respecto, Herrera,
(1997) no encontró diferencias en la producción inicial según el mes de inicio de la lactancia al evaluar dos predios lecheros del sur de Chile.

En lactancias de primavera, a diferencia de lo observado con lactancias de otoño, la producción inicial así como el "peak" de la curva se hace mayor mientras más tardío es el parto. Esto indicaría que las vacas paridas en julio no expresan su potencial al inicio de la lactancia, siendo deseable que vacas de partos más tempranos comiencen con una producción inicial similar a las de pariciones más tardías. La razón de estas diferencias también estaría en un efecto combinado de la alimentación preparto y la contribución de reservas corporales. Es claro que en el caso de lactancias iniciadas en julio, y en menor grado, de las iniciadas en agosto, ha existido un desgaste de reservas previa al mes de octubre, asociado con una menor calidad de los forrajes conservados en comparación con la pradera, siendo decreciente la contribución de las reservas corporales mientras más temprano ha sido el parto. Por lo tanto, si todas las vacas parieran en julio y agosto, debería existir una suplementación, la cual, además de influir en una mayor producción de leche al inicio de la lactancia, ayudaría en una mayor eficiencia reproductiva toda vez que las vacas lleguen en un estado más avanzado a la etapa de encaste. Esto es de gran importancia, ya que si las vacas se atrasan en entrar en celo, cada año las pariciones van ocurriendo más tarde (González, 1995; González y Magofke, 2003).

Dadas las condiciones de crecimiento de la pradera en la zona sur de Chile, un atraso en la época de parto, además del efecto negativo en la producción por lactancia, puede retrasar de manera marcada la recuperación de peso de las vacas.

La producción posterior, sin embargo, decrece de manera inversa con el nivel de producción al "peak", asociada a una menor persistencia, que tiende a mantenerse hasta el final de la lactancia (Cuadro 1, Figura 3). Por lo tanto, la menor producción inicial por parte de vacas de parición temprana (julio, agosto), fue ampliamente compensada por la mayor producción posterior. Esta mayor producción estaría explicada por una alta disponibilidad de praderas de buena calidad durante el primer mes de lactancia, acompañada por una mejor condición corporal al parto como consecuencia de una mejor alimentación previa al parto. Lo anterior demuestra 
que el deterioro de calidad de la pradera que se produce al avanzar la primavera y durante el verano, afecta más en el caso de las lactancias iniciadas más tardíamente (Magofke et al., 1984; Herrera, 1997; García et al., 1999). De este modo, en las vacas confinadas, debido a la limitante nutricional antes descrita, no se expresó la correlación positiva existente entre producción al "peak" y producción total. Dicha asociación sólo se manifestará cuando las lactancias se caractericen por un marcado incremento en la producción posparto y una declinación gradual después de alcanzado el "peak".

Debido a la similitud en la composición de la leche, independientemente del mes de parto dentro de época, las diferencias en producción de grasa y proteína responden esencialmente a diferencias en la producción de leche por lactancia. Los resultados obtenidos en predios de la zona (Magofke et al., 1984; Cárdenas, 1994; García et al., 1999), para vacas de parto primaveral se asemejan bastante a lo expuesto en este estudio.

En consecuencia, el mayor impacto productivo para una explotación biestacional se lograría por un conjunto de medidas entre las cuales destacan: el adelantamiento de los partos en ambas épocas; el aumento de producción de las vacas de parto de otoño durante la primera mitad de la lactancia, por una mejora en la alimentación invernal; y un aumento de producción de las vacas de parto de primavera, principalmente durante la segunda mitad de la lactancia, a través de tecnologías que permitan una mayor disponibilidad y calidad del forraje.

\section{CONCLUSIONES}

Bajo las condiciones de manejo imperantes en el sistema evaluado, en que coexisten partos de primavera y de otoño, se comprobó una mayor producción con lactancias iniciadas en otoño, aunque las diferencias no fueron marcadas.

Durante la primera mitad de la lactancia, la producción fue menor en partos de otoño que con partos de primavera. Contrariamente, en la segunda mitad de la lactancia, la producción fue menor en partos de primavera que en partos de otoño. En ambos casos, esto sería indicativo de una subutilización del potencial productivo de las vacas por razones nutricionales.

Las curvas de lactancia en los períodos de pastoreo estuvieron fuertemente influenciadas por el crecimiento de la pradera, efecto que fue muy marcado en la lactancia inicial de las vacas paridas en primavera y en el último tercio de la lactancia de vacas de otoño, especialmente con partos tardíos.

En ambas épocas, los partos más tempranos se asocian a mayores niveles productivos debido a que lograron lactancias más largas y persistentes.

Aunque hubo una mayor concentración de proteína láctea con partos de primavera, las concentraciones de proteína y grasa no variaron sustancialmente entre épocas y mes de parto dentro de época. Por consiguiente la producción de sólidos siguió una tendencia similar a la descrita para producción láctea.

\section{LITERATURA CITADA}

Anrique, R., O. Balocchi, L. Latrille, D. Pinochet, D. Alomar, V. Moreira, et al. 1999. Competitividad de la producción lechera nacional. Vol. 1. 216 p. Universidad Austral de Chile, Facultad de Ciencias Agrarias, Valdivia, Chile.

Anrique, R., L. Latrille, O. Balocchi, D. Pinochet, V. Moreira, R. Smith, et al. 2004. La producción de leche en Chile: caracterización técnica a nivel predial. 59 p. Universidad Austral de Chile, Facultad de Ciencias Agrarias, Valdivia, Chile.
Bravo, M. 1998. Efectos de diferentes grados de cruzamientos con el genotipo Holstein Friesian sobre la producción y curva de lactancia en la lechería Punahue. 109 p. Tesis Ingeniero Agrónomo. Universidad Austral de Chile, Facultad de Ciencias Agrarias, Valdivia, Chile.

Cárdenas, C. 1994. Evaluación genética y ambiental de la producción de leche y grasa en el rebaño de la Estación Experimental Oromo (Holstein, Holando Europeo). 71 p. Tesis Ingeniero Agrónomo. Universidad de Chile, Facultad de Ciencias Agrarias, Santiago, Chile. 
Cortés, C. 1986. Uso de registros parciales de producción de leche como criterio de selección en un sistema de parición estacional de primavera en la X Región. 71 p. Tesis Ingeniero Agrónomo. Universidad de Chile, Facultad de Ciencias Agrarias y Forestales, Santiago, Chile.

García, X., H. González, J. Magofke, C. Cortés, y C. Cárdenas. 1999. Efecto del año y mes de parto sobre la producción de leche y grasa, en un rebaño de vacas de la X Región sometido a cambios de manejo. Avances en Producción Animal 24 (1-2):121-131.

García, X., J. Magofke, H. González, y C. Cortés. 1987. Registros parciales de producción de leche como criterio de selección en vacas Holando europeo. I. Efecto de la edad, año y mes de parición sobre la producción total y parcial de leche. Avances en Producción Animal 12(1-2):111-124.

García, X., J. Magofke, E. Riveros, y C. Hepp. 1984. Factores no genéticos que influyen sobre la producción de leche y materia grasa de vacas Holando europeo, en un sistema con pariciones estacionales en la X Región. II. Efecto de la edad, número del parto, período de servicio y período seco. Avances en Producción Animal 9(1-2):99-108.

González, H. 1995. Cuantificación de los efectos que determinan el comportamiento reproductivo en un rebaño lechero con parición estacional en la X Región. 117 p. Tesis M.Sc. Universidad Austral de Chile, Facultad de Ciencias Agrarias, Valdivia, Chile.

González, H. 2002. Factores nutricionales que afectan la producción y composición de la leche. Circular de Extensión. N ${ }^{\circ} 28$ p.12-21. Universidad de Chile, Depto. de Producción Animal, Santiago, Chile.

González, H., y J. Magofke. 1994. Evolución del comportamiento productivo y reproductivo del plantel lechero de la Estación Experimental Oromo. Avances en Producción Animal (B-18):66-97.

González, H., y J. Magofke. 2003. Comportamiento reproductivo en rebaños lecheros con partos estacionales. Avances en Producción Animal 28(1-2):3-13.

Herrera, J. 1997. Evaluación de algunos efectos genéticos y no genéticos sobre el comportamiento productivo, en dos rebaños lecheros de la X Región. 150 p. Tesis M.Sc. Universidad Austral de Chile, Facultad de Ciencias Agrarias, Valdivia, Chile.

Holmes, C., I. Brookes, D. Garrick, D. Mackenzie, T. Parkinson, and G. Wilson. 2002. Milk production from pasture. 602 p. Massey University, Palmerston North, New Zealand.
Lasley, J. 1982. Genética del mejoramiento del ganado. 341 p. UTEHA, México.

Latrille, L. 1985. La curva de lactancia. Algunos factores que afectan la cantidad y la composición de la leche. Las reservas corporales al momento del parto; la predicción del consumo voluntario. Alimentación de bovinos para producción de leche y carne. (B-10) p. 12-39. Universidad Austral de Chile, Instituto de Producción Animal, Valdivia, Chile.

Latrille, L. 1993. El valor nutritivo de la leche bovina y factores que alteran su composición. Universidad Austral de Chile, Instituto de Producción Animal (B17):27-56.

Letelier, A. 1998. Composición de la leche cruda de la VIII, IX y X Regiones. 70 p. Tesis Ingeniero Agrónomo. Universidad Austral de Chile, Facultad de Ciencias Agrarias, Valdivia, Chile.

Magofke, J., X. García, E. Riveros, y C. Hepp. 1984. Factores no genéticos que influyen sobre la producción de leche y materia grasa de vacas Holando europeo, en un sistema con pariciones estacionales en la X Región. I. Efecto del año y mes de parto. Avances en Producción Animal 9(1-2):83-97.

O’Brien, B., J. Murphy, J. Connolly, R. Mehra, T. Guinee, and G. Stakelum. 1997. Effect of altering the daily herbage allowance in mid lactation on the composition and processing characteristics of bovine milk. J. Dairy Res. 64:621-626.

Pinto, M., E. Carrasco, B. Fraser, A. Letelier, y P. Dörner. 1998. Composición química de la leche cruda y sus variaciones a nivel de silos en plantas lecheras de la VII, IX y X Regiones de Chile. Parte I. Macrocomponentes. Agro Sur (Chile) 26(2):97-109.

Riveros, E., y J. Magofke. 1983. Influencia de la estacionalidad de la producción de una pradera naturalizada húmeda sobre la producción de leche de vacas holando-europeas según la época de parto. Avances en Producción Animal 8(1-2):37-49.

SAS Institute. 1992. STAT Guide for personal computers. 704 p. $8^{\text {th }}$ ed. SAS Institute Inc., Cary, North Carolina, USA.

Teuber, N., y O. Balocchi. 2003. Recursos forrajeros en producción de leche. I. Balance alimenticio con los recursos del sur. Seminario: Hagamos de la lechería un mejor negocio. Serie Actas INIA No 24 p. 3-11. 